\title{
Avaliação espacial e temporal de aspectos sanitários de reservatórios com captação de água para abastecimento em SP com ênfase em cianobactérias e cianotoxinas
}

Spatial and temporal assessment of sanitary aspects of public water supply reservoirs in SP, Brazil, with emphasis on cyanobacteria and cyanotoxins

\author{
Henrique Gamon Sonobe ${ }^{1} \odot$, Marta Condé Lamparelli² ${ }^{\oplus}$, Davi Gasparini Fernandes Cunha** ${ }^{1 *}$
}

\begin{abstract}
RESUMO
A presença de cianobactérias e cianotoxinas em reservatórios no estado de São Paulo constitui um desafio para produção de água potável. Sete reservatórios utilizados para abastecimento público, monitorados pela Companhia Ambiental do Estado de São Paulo (CETESB), foram analisados em relação a cianobactérias, microcistina e indicadores de contaminação fecal entre 2011 e 2015. Calcularam-se porcentagens de não conformidade com padrões de qualidade. Os resultados evidenciaram a presença de cianobactérias e microcistina (máximo de 22,4 $\mu \mathrm{g} . \mathrm{L}^{\mathrm{L}}$ ), além de conflitos com os padrões da Resolução CONAMA no 357/2005 (BRASIL, 2005), o que reforça a importância de investimentos em coleta e tratamento de esgotos sanitários. É discutida a necessidade de maior integração entre as leis e resoluções que versam sobre qualidade da água, além do melhor planejamento dos recursos hídricos, com foco em metas progressivas de melhoria da qualidade da água. Palavras-chave: reservatórios; esgoto sanitário; cianotoxinas; abastecimento público; estado de São Paulo.
\end{abstract}

\begin{abstract}
The occurrence of cyanobacteria and cyanotoxins in reservoirs in São Paulo State is a challenge to the production of drinking water. Seven reservoirs used for public supply and monitored by CETESB, were analyzed regarding cyanobacteria, microcystin and indicators of fecal contamination between 2011-2015. The percentages of non-compliance with water quality standards were calculated. Results highlighted the presence of cyanobacteria and microcystin (maximum of $22.4 \mu \mathrm{g} / \mathrm{L}$ ), as well as conflicts with the standards provided by CONAMA Resolution No. 357/2005 (BRASIL, 2005), which reinforces the importance of investments in sanitary sewage collection and treatment. The need for greater integration between laws and resolutions on issues related to water quality were also discussed, as well as better planning of water resources, focusing on progressive goals for water quality improvement.
\end{abstract}

Keywords: reservoirs; sanitary sewage; cyanotoxins; public supply; São Paulo State.

\section{INTRODUÇÃO}

A qualidade da água bruta dos sistemas aquáticos tem se agravado globalmente em função da poluição gerada pelas atividades antropogênicas e das formas inadequadas de uso e ocupação do solo, com repercussões negativas sobre os usos múltiplos da água e a proteção da biodiversidade (VÖRÖSMARTY et al., 2010; CUNHA et al., 2016). No Brasil, o déficit de sistemas de esgotamento sanitário (IBGE, 2008; ANA, 2013; PAIVA; SOUZA, 2018) é uma das maiores preocupações por sua influência sobre a qualidade da água de mananciais de abastecimento público, especialmente de reservatórios, que são uma alternativa recorrente como manancial de captação de água para consumo humano no país. O aporte de poluentes de fontes difusas e de efluentes brutos ou não adequadamente tratados diminui a qualidade dos mananciais (CUNHA et al., 2011), o que se reflete em diminuição das concentrações de oxigênio dissolvido no meio, entre outras alterações indesejáveis que comprometem os serviços ecossistêmicos (DODDS; PERKIN; GERKEN, 2013). Consequentemente, podem ocorrer prejuízos à 
saúde pública pela presença de substâncias tóxicas (e.g., metais e cianotoxinas) e ocorrência de organismos patogênicos.

Além do aporte de esgoto sanitário, a lixiviação de fertilizantes de áreas agrícolas tem contribuído para a maior disponibilidade de nutrientes nos reservatórios e consequentemente aumento do seu estado trófico (CUNHA; CALIJURI, 2011b). A presença de nutrientes, sobretudo nitrogênio e fósforo, aliada a condições ambientais como temperatura e disponibilidade de luz, pode favorecer a proliferação de cianobactérias (MEREL et al., 2013). Os estudos relacionados a esses microrganismos têm despertado especial interesse nos últimos anos, sobretudo por algumas espécies serem capazes de produzir toxinas, como as microcistinas, frequentemente reportadas na literatura (e.g., LOFTIN et al., 2016; WOOD, 2016). No Brasil, diversos são os relatos de presença de cianobactérias em reservatórios (HIROOKA et al., 1999; CHELLAPPA; COSTA; MARINHO, 2000; MATTHIENSEN et al., 2000; CARVALHO et al., 2007; GUEDES et al., 2014; LOPES et al., 2017), e alguns destacam os agravos à saúde causados por esses microrganismos (TEIXEIRA et al., 1993; AZEVEDO et al., 2002). As cianotoxinas podem ter efeitos neuro, hepato ou dermatotóxicos e levar até mesmo à morte (SVIRČEV et al., 2017).

A produção de água potável vem se tornando um desafio em função da degradação da qualidade dos mananciais. O relatório Qualidade das Águas Interiores no Estado de São Paulo de 2017 (CETESB, 2017), utilizando o Índice de Qualidade de Águas para Abastecimento Público (IAP), apontou que, entre 90 pontos de captação monitorados, $18 \%$ possuíam qualidade anual ruim ou péssima e outros 34\% foram classificados como regulares. Cunha et al. (2013) relataram que muitos sistemas aquáticos no estado de São Paulo apresentam conflitos com o seu enquadramento e os padrões fixados pela Resolução CONAMA $n^{\circ}$ 357/2005 (BRASIL, 2005).

Entender como ocorrem florações de cianobactérias e o que favorece a produção de toxinas é importante para a melhor gestão dos recursos hídricos. Moschini-Carlos et al. (2009) estudaram a qualidade da água do reservatório Billings (braço do Taquacetuba), usado para abastecimento público por meio da transposição para a Represa Guarapiranga. Os pesquisadores relataram a presença de cianotoxinas na água em diferentes estações do ano, como as microcistinas (MCRR, MC-LR e MC-YR). Além disso, observaram que a densidade de cianobactérias foi maior em condições de baixa relação nitrogênio/ fósforo e sob temperaturas amenas $\left(\sim 19,5^{\circ} \mathrm{C}\right)$. Embora existam estudos como esse, ainda são necessárias mais informações sobre como a concentração de cianotoxinas e demais características relevantes de mananciais de abastecimento público variam espacial e temporalmente em SP, estado mais populoso do Brasil e com demanda significativa de água para diversos usos.

O objetivo da presente pesquisa foi avaliar variáveis da água de relevância sanitária em sete reservatórios utilizados para captação de água bruta para abastecimento público monitorados pela Companhia Ambiental do Estado de São Paulo (CETESB) nesse estado. Para isso, foram definidos os seguintes objetivos específicos:

- investigar a variação sazonal e espacial de cianobactérias, microcistina e indicadores de contaminação fecal na água;

- identificar relações entre algumas características limnológicas dos reservatórios e a presença de cianobactérias/cianotoxinas;

- determinar a ocorrência de conflitos entre os resultados do monitoramento de tais sistemas aquáticos e os seus respectivos enquadramentos, com base nos padrões ambientais estabelecidos pela legislação vigente.

\section{METODOLOGIA}

A partir da compilação de dados disponíveis no sistema InfoÁguas da CETESB (https://servicos.cetesb.sp.gov.br/infoaguas/), foram analisadas variáveis de qualidade da água [número de cianobactérias, concentração de microcistinas, clorofila-a, fósforo total, nitrogênio amoniacal, nitrato, demanda bioquímica de oxigênio (DBO), coliformes termotolerantes, E. coli e temperatura] em pontos de monitoramento em sete reservatórios no estado de São Paulo (SP) (Tabela 1). As metodologias analíticas estão detalhadas no Apêndice E de CETESB (2017). A avaliação considerou dados pareados relativos ao período entre 2011 e 2015, com frequência bimestral de monitoramento. Entre esses sistemas aquáticos, os reservatórios de Itupararanga e Cascata são enquadrados como classe 2 e os demais, como classe especial. Além disso, a maioria dos reservatórios estudados se situa em unidades de gerenciamento de recursos hídricos (UGRHI) classificadas, segundo a CETESB, como industriais, com exceção do Cascata, cuja aptidão da UGRHI é para a agropecuária.

Os resultados de qualidade da água foram comparados aos padrões estabelecidos pela Resolução CONAMA n 357/2005 (BRASIL, 2005), e os reservatórios da classe especial foram comparados com os valores estabelecidos para a classe 1 , pois não há critérios específicos para essa categoria. Segundo o artigo 13 da CONAMA no 357/2005 (BRASIL, 2005), nas águas de classe especial, deverão ser mantidas as condições naturais do corpo de água. A classe 1 é o padrão mais restritivo entre as classes estabelecidas e, portanto, possui os parâmetros que mais se assemelhariam às condições naturais do corpo d'água. Também foram utilizados como referência os padrões de potabilidade estabelecidos pela Portaria de Consolidação no 5/GM/MS (BRASIL, 2017). No entanto, deve-se ressaltar que a frequência de monitoramento dos dados disponíveis no sistema InfoÁguas da CETESB (bimestral) e a frequência exigida pela portaria supracitada (em alguns casos, semanal) são diferentes, mas ainda assim a comparação foi considerada válida. A relação clorofila-a/microcistina foi calculada para todos os reservatórios. Ao se calcular tal razão, procurou-se avaliar o potencial de produção dessa cianotoxina em relação à biomassa presente. 
Como os dados apresentaram distribuição não normal, foram realizados testes estatísticos de Kruskal-Wallis, a um nível de confiança de $95 \%$ ( $p<0,05)$, para os fatores temporal (diferentes anos) e espacial (diferentes reservatórios) com o objetivo de verificar suas influências sobre as concentrações de microcistina, número de cianobactérias e concentração de coliformes e E. coli. O teste de Spearman foi utilizado para verificar as correlações das variáveis de qualidade de água entre si.

\section{RESULTADOS E DISCUSSÃO}

Os reservatórios apresentaram-se predominantemente eutrofizados durante o período analisado, segundo o Índice de Estado Trófico (IET), divulgado pelo Relatório de Qualidade das Águas Interiores de São Paulo de 2017 (CETESB, 2017). Entre os anos de 2011 e 2015, as maiores concentrações médias de fósforo total (Tabela 2) foram observadas nos reservatórios Billings e Guarapiranga (0,06 e 0,10 mg.L $\mathrm{L}^{-1}$, respectivamente). As concentrações das formas nitrogenadas variaram de
0,15 a 0,33 mg. $\mathrm{L}^{-1}$ para nitrogênio amoniacal e de 0,19 a $0,81 \mathrm{mg} \cdot \mathrm{L}^{-1}$ para nitrato, com as maiores concentrações nos reservatórios Jaguari, Cascata e Guarapiranga. As temperaturas médias das águas dos reservatórios foram semelhantes, apresentando valores próximos aos $23^{\circ} \mathrm{C}$. Da mesma forma, os dados de DBO apresentaram variação relativamente pequena, com médias entre 3 e 6 mg. $\mathrm{L}^{-1}$.

Concentrações iguais ou maiores que $50 \mu \mathrm{g} . \mathrm{L}^{-1}$ de clorofila-a, quando associadas a florescimentos tóxicos de cianobactérias e presença de cianotoxinas, são condições que podem deflagrar o alerta nível 2 na árvore de decisão proposta por Chorus e Bartram (1999). Nesse sentido, a concentração média de clorofila-a observada no reservatório Billings $\left(89,9 \pm 80,1 \mu \mathrm{g} . \mathrm{L}^{-1}\right.$, Tabela 2$)$ seria elevada o suficiente para considerar esse alerta. Segundo as diretrizes propostas pelos autores, quando as estações de tratamento de água não dispõem de tratamento avançado, como carvão ativado, um plano de contingência deve ser acionado, o que inclui comunicação à comunidade por meio da mídia, abastecimento de água por meio de um manancial alternativo e até mesmo

Tabela1 - Reservatórios do estado de São Paulo selecionados na presente pesquisa, incluindo nome, ponto de monitoramento da Companhia Ambiental do Estado de São Paulo, coordenadas geográficas, área, tempo de residência, município a que pertencem, além de sua classe de enquadramento.

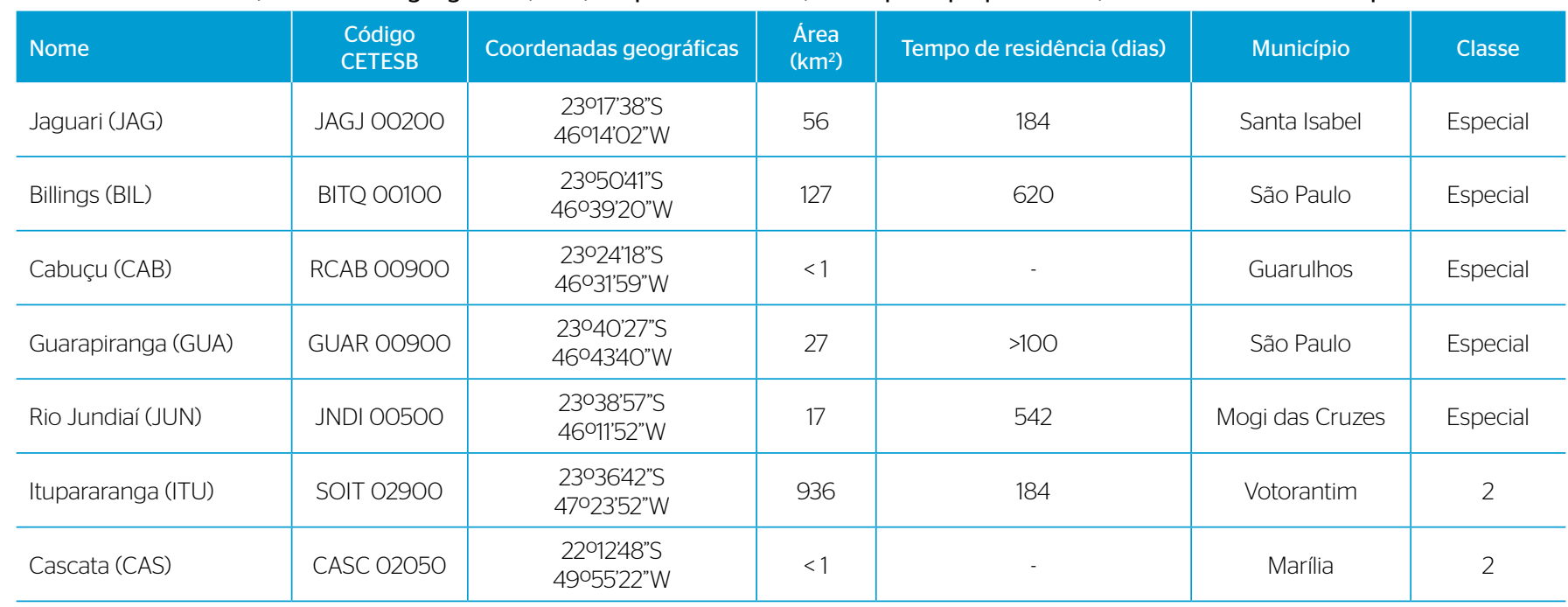

: Informação não disponível.

Fonte: adaptado de Cunha et al. (2013) e Cetesb (2017).

Tabela 2 - Médias e desvios padrão das variáveis de qualidade da água nos reservatórios estudados no Estado de São Paulo no período de 2011 a 2015.

\begin{tabular}{l|c|c|c|c|c|c}
\hline Reservatório & Clorofila-a $\left(\mu \mathrm{g} \cdot \mathrm{L}^{-1}\right)$ & $\mathrm{PT}\left(\mathrm{mg} \cdot \mathrm{L}^{-1}\right)$ & $\mathrm{N}$-amon $\left(\mathrm{mg} \cdot \mathrm{L}^{-1}\right)$ & $\mathrm{NO}_{3}\left(\mathrm{mg} \cdot \mathrm{L}^{-1}\right)$ & DBO $\left(\mathrm{mg} \cdot \mathrm{L}^{-1}\right)$ & Temperatura $\left({ }^{\circ} \mathrm{C}\right)$ \\
\hline JAG & $42,3 \pm 81,3$ & $0,05 \pm 0,06$ & $0,33 \pm 0,21$ & $0,22 \pm 0,22$ & $5 \pm 5$ & $22,6 \pm 4,2$ \\
\hline BIL & $89,9 \pm 80,1$ & $0,06 \pm 0,03$ & $0,15 \pm 0,08$ & $0,27 \pm 0,12$ & $6 \pm 4$ & $22,8 \pm 3,7$ \\
\hline ITU & $14,6 \pm 4,7$ & $0,01 \pm 0,01$ & $0,18 \pm 0,07$ & $0,19 \pm 0,03$ & $3 \pm 2$ & $22,8 \pm 3,5$ \\
\hline CAS & $43,5 \pm 35,3$ & $0,03 \pm 0,02$ & $0,33 \pm 0,29$ & $0,54 \pm 0,36$ & $5 \pm 2$ & $23,6 \pm 3,9$ \\
\hline CAB & $11,2 \pm 11,0$ & $0,03 \pm 0,01$ & $0,24 \pm 0,08$ & $0,22 \pm 0,07$ & $3 \pm 1$ & $23,2 \pm 3,5$ \\
\hline GUA & $34,7 \pm 16,4$ & $0,10 \pm 0,24$ & $0,27 \pm 0,21$ & $0,81 \pm 0,26$ & $4 \pm 1$ & $22,6 \pm 3,3$ \\
\hline JUN & $29,1 \pm 18,9$ & $0,04 \pm 0,02$ & $0,16 \pm 0,08$ & $0,20 \pm 0,01$ & $4 \pm 2$ & $22,8 \pm 4,0$ \\
\hline
\end{tabular}

PT: fósforo total; N-amon: nitrogênio amoniacal; $\mathrm{NO}_{3}$ : nitrato; $\mathrm{DBO}$ : demanda bioquímica de oxigênio; JAG: Jaguari; BIL: Billings; ITU: Itupararanga; CAS: Cascata; CAB: Cabuçu: GUA: Guarapiranga; JUN: rio Jundiaí. 
fornecimento de água engarrafada para os consumidores. Entre os reservatórios com menores concentrações de clorofila, destacaram-se Itupararanga e Cabuçu, com médias inferiores a $15 \mu \mathrm{g} . \mathrm{L}^{-1}$.

\section{Variação sazonal e espacial de cianobactérias, microcistina e indicadores de contaminação fecal na água}

As maiores densidades médias de cianobactérias foram observadas nos reservatórios Billings, Itupararanga, Cascata e Guarapiranga, respectivamente, em células. $\mathrm{mL}^{-1}: 277.132,169.671,177.996$ e 50.247 (Figura 1A). Tais resultados indicam que a ocorrência desses microrganismos é comum e que as densidades frequentemente ultrapassaram o limite de 50.000 células. $\mathrm{mL}^{-1}$ estabelecido pela Resolução CONAMA n 357/2005 (BRASIL, 2005) para corpos d'água de classe 2.

Os outliers observados na Figura 1B sugerem a ocorrência de florações tóxicas e presença de microcistina, principalmente nos reservatórios Billings e Cascata, cujas concentrações máximas atingiram 22,4 e 10,9 $\mu \mathrm{g} . \mathrm{L}^{-1}$, respectivamente. Além desses, apenas no reservatório Jaguari foram observadas concentrações acima de $1 \mu \mathrm{g} . \mathrm{L}^{-1}$, considerada referência de microcistina para água tratada pelo Anexo XX da Portaria de Consolidação no 5/GM/MS (BRASIL, 2017), que estabeleceu padrões de potabilidade nacionais. Embora as águas brutas, de acordo com a legislação mencionada, não representem água tratada, os valores elevados observados alertam para a possibilidade de intoxicação da população, uma vez que a remoção da toxina depende da capacidade do processo de tratamento de água, sendo que tratamentos convencionais podem não ter a eficácia necessária (HIMBERG et al., 1989; HALL et al., 2000).

Os dados de coliformes termotolerantes e E. coli (Figura 1C) no reservatório Cascata sugerem contaminação por esgoto sanitário. A contaminação por efluentes domésticos e o consequente aporte de nutrientes para o corpo d'água podem contribuir para as elevadas densidades de cianobactérias e concentrações microcistina nesse reservatório. Embora contaminadas, suas águas apresentam valores de coliformes termotolerantes menores que o limite para as águas com qualidade "satisfatória" para recreação de contato primário, segundo a Resolução CONAMA n 274/2000 (BRASIL, 2000). No entanto, essa classificação não pode ser adotada, uma vez que a frequência dos dados obtidos no sistema InfoÁguas, da CETESB, foi bimestral, e não semanal. A resolução citada define que $80 \%$ de um conjunto de amostras obtidas em cada 1 das 5 semanas anteriores, colhidas no mesmo local, estejam abaixo de 1.000 UFC. $100 \mathrm{~mL}^{-1}$ para serem classificadas como "satisfatórias". Com essa mesma ressalva, nos demais reservatórios, a qualidade das águas pôde ser comparada ao considerado como "excelente" pela mesma resolução (menos de 250 UFC de coliformes termotolerantes. $100 \mathrm{~mL}^{-1}$ ) em relação à contaminação por esgoto sanitário.
O desenvolvimento de cianobactérias nos reservatórios do estado de São Paulo apresentou-se estável ao longo dos anos (Figura 1D), assim como as concentrações de microcistina (Figura 1E) e coliformes termotolerantes (Figura 1F). No entanto, os outliers presentes na Figura 1E podem indicar florações mais tóxicas no último ano avaliado (2015).

A densidade de cianobactérias, a produção de microcistina e a concentração de coliformes termotolerantes pareceram ser mais afetadas pelas características de cada reservatório em comparação à variação interanual dos dados (Tabela 3). O teste de Kruskal-Wallis com as variáveis número de células de cianobactérias, microcistina e E. coli sugeriu que o fator espacial (diferentes reservatórios) foi mais significativo que o temporal (diferentes anos). Esse resultado reforça a importância do disciplinamento do uso e da ocupação do solo para a conservação da qualidade dos corpos hídricos.

\section{Relações entre características limnológicas dos reservatórios e a proliferação de cianobactérias e a produção de cianotoxinas}

As cianobactérias possuem clorofila-a como um de seus pigmentos fotossintéticos (CALIJURI; ALVES; SANTOS, 2006), a qual indicar indireta e quantitativamente a presença desses microrganismos ou de outros grupos fitoplanctônicos, de maneira geral. Foi observada correlação significativa entre a densidade de cianobactérias e a clorofila-a $(\rho=0,535$, Spearman, Tabela 4). Houve ainda correlação significativa, positiva ou negativa, com DBO $(\rho=0,183)$, nitrogênio amoniacal $(\rho=-0,309)$ e coliformes termotolerantes e E. coli $(\rho=-0,413)$. Embora alguns estudos comumente apontem que o fósforo costuma ser o nutriente limitante para a produção primária (CORRELL, 1999; SCHINDLER et al., 2008), especialmente em ambientes tropicais, ele não foi correlacionado com a densidade de cianobactérias. A colimitação por fósforo e nitrogênio (ELSER et al., 2007) também tem sido descrita como uma possibilidade em alguns reservatórios tropicais (CUNHA; CALIJURI, 2011a).

A correlação inversa entre a densidade de cianobactérias e as concentrações de nitrogênio amoniacal também foi observada por Kotak et al. (2000). Os autores apontaram que a necessidade de nitrogênio varia de acordo com a espécie de cianobactéria. Como a correlação com outras formas de nitrogênio não foi significativa (nitrato $\rho=0,091 \mathrm{e}$ nitrito $\rho=0,005)$ e as concentrações médias de nitrogênio amoniacal estiveram abaixo do limite estabelecido para a classe 2, é possível supor que as cianobactérias presentes nos reservatórios estudados provavelmente são capazes de crescer em ambientes com baixa concentração de nitrogênio e/ou possuem a capacidade de fixar o nitrogênio atmosférico. De fato, o relatório da CETESB (2017) reportou os gêneros de cianobactérias mais comuns nos reservatórios, entre eles potenciais fixadores de $\mathrm{N}_{2}$. Alguns exemplos são: Aphanizomenon (Jaguari), Cylindrospermopsis (Jaguari, Billings e Itupararanga) e Aphanocapsa (Guarapiranga e Rio Jundiaí). 
Já a microcistina foi correlacionada com o número de células de cianobactérias $(\rho=0,455)$ e clorofila-a $(\rho=0,387)$, indicando que as cianobactérias que ocorrem nos reservatórios estudados são potenciais produtoras dessa toxina. Além disso, a correlação com o fósforo, embora moderada $(\rho=0,232)$, sugere que esse elemento influencia positivamente a produção da cianotoxina. Na literatura, essa influência é discutida.

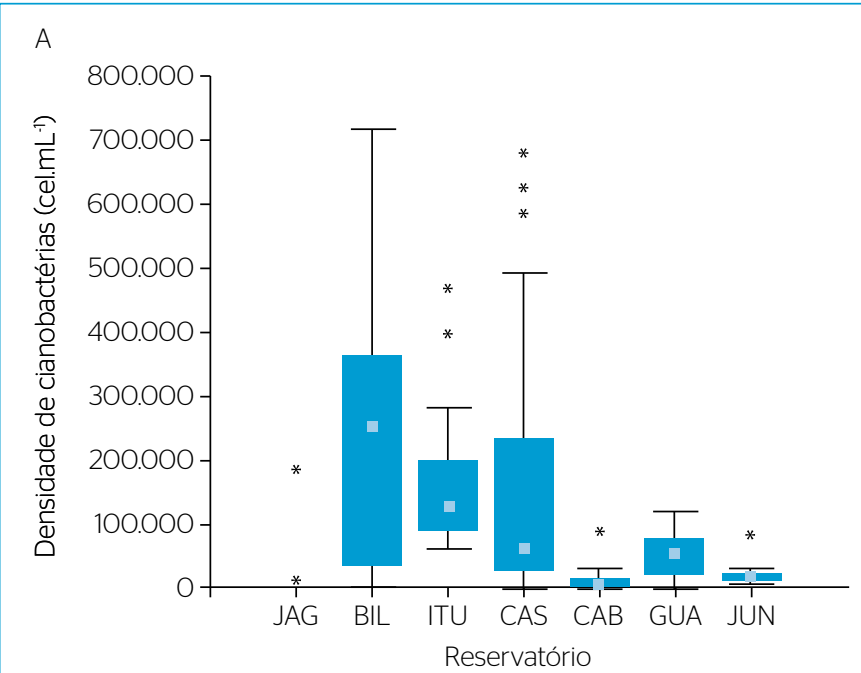

B

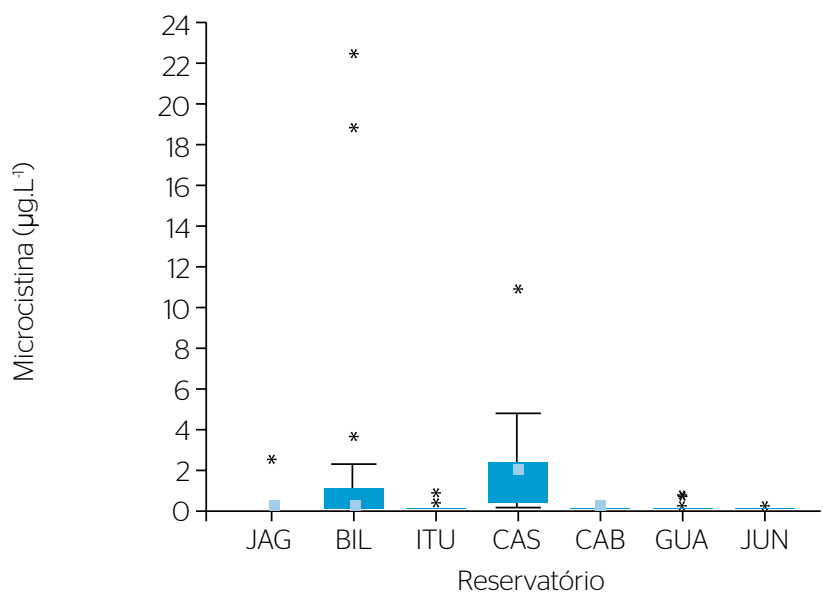

C

D
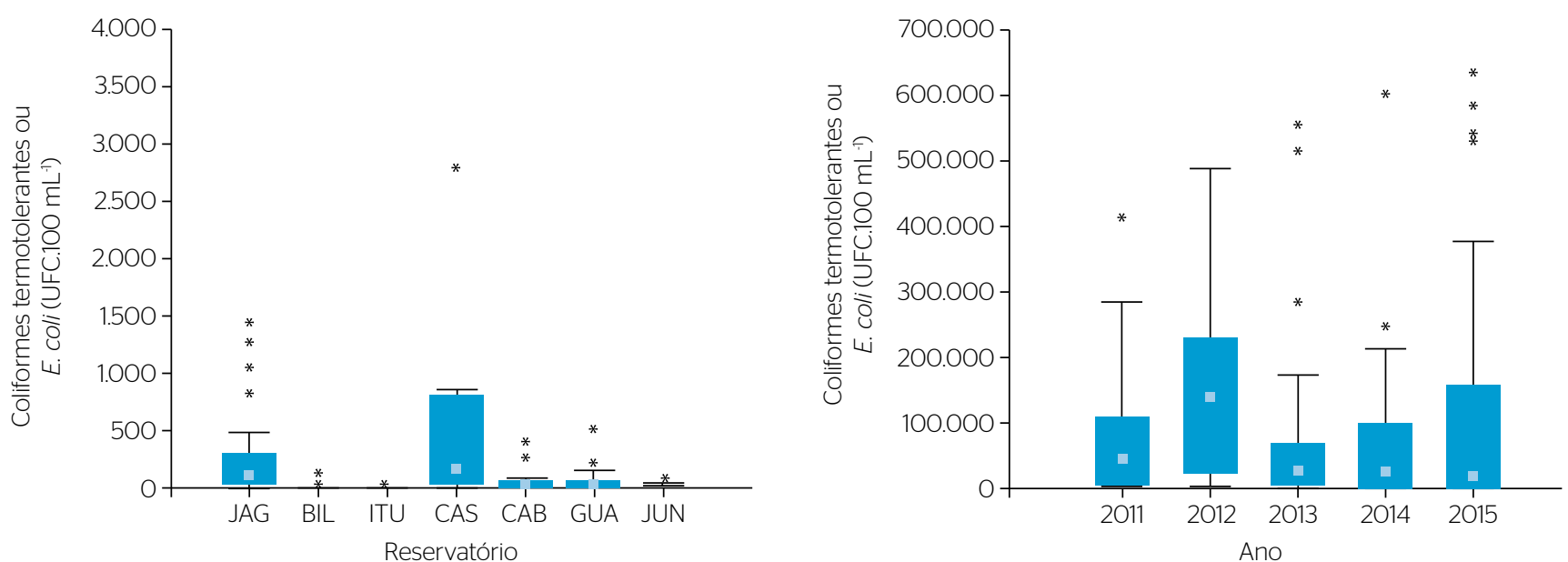

$\mathrm{E}$

F
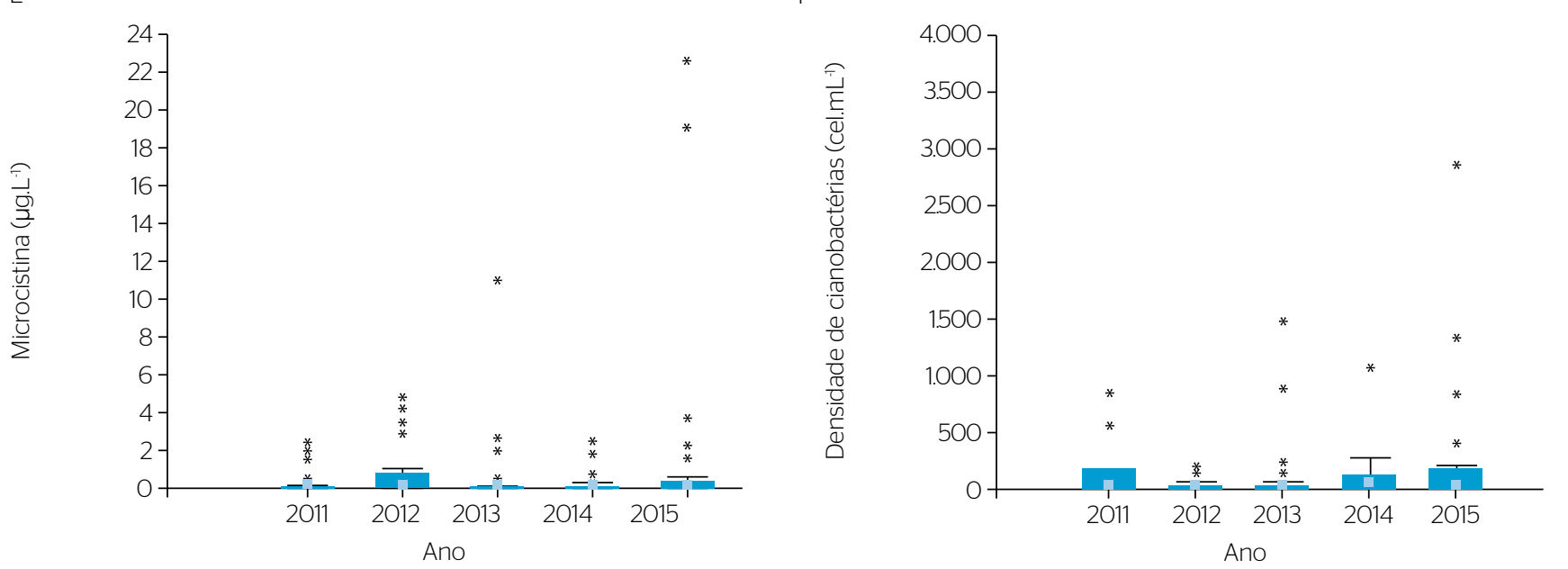

Figura 1 - Variação espacial e temporal do número de células de cianobactérias, microcistina e coliformes termotolerantes ou Escherichia coli. 
Kotak et al. (2000) observaram que a concentração de microcistina produzida pela espécie Microcystis aeruginosa aumenta em ambientes ricos em fósforo. No entanto, Oh et al. (2000) relataram que a produção de toxina é maior em ambientes com limitação de fósforo. No presente estudo, foi encontrada ainda correlação moderada da cianotoxina com a DBO $(\rho=0,258)$, a qual, assim como o fósforo, é um parâmetro influenciado por descargas de esgotos sanitários.

Contrariamente ao que foi observado com as cianobactérias, que não apresentaram correlação significativa com o fósforo total, a clorofila-a foi correlacionada positivamente com esse nutriente $(\rho=0,438)$, indicando que os demais grupos fitoplanctônicos podem ser favorecidos por ele. A clorofila-a foi ainda correlacionada negativamente com nitrogênio amoniacal $(\rho=-0,261)$ e positivamente com a DBO ( $\rho=$ $0,648)$. As concentrações de nitrito e nitrato, assim como a temperatura, aparentemente não influenciaram a densidade de cianobactérias, a concentração de microcistina e a de clorofila-a.

Ao considerar a razão entre as concentrações de microcistina e de clorofila-a, foi possível verificar que o potencial tóxico das águas dos reservatórios tem apresentado tendência de aumento desde 2011, com destaque para o ano de 2015 (Figura 2). Nesta análise, os reservatórios Cascata e Jaguari apresentaram as maiores variações. No entanto,

Tabela 3 - Resultado do teste estatístico de Kruskal-Wallis para verificar a influência dos fatores temporais e espaciais sobre as concentrações de microcistina, número de células de cianobactérias e concentração de coliformes e Escherichia coli.

\begin{tabular}{l|c|c|c} 
Fator & $\begin{array}{c}\text { Número de células } \\
\text { de cianobactérias }\end{array}$ & Microcistina & $\begin{array}{c}\text { Coliformes termo- } \\
\text { tolerantes ou } E \text {. coli }\end{array}$ \\
\hline Reservatório & $p=0,0001^{1}$ & $p=0,009$ & $p=0,0261$ \\
\hline Ano & $p=0,151$ & $p=0,326$ & $p=0,307$ \\
\hline
\end{tabular}

' $p<0,05$ (significativo a um nível de confiança de 95\%).

Tabela 4 - Correlações entre as variáveis limnológicas e o número de células de cianobactérias, concentrações de microcistina e clorofila-a.

\begin{tabular}{l|c|c|c} 
& Cianobactérias & Microcistina & Clorofila-a \\
\hline Cianobactérias & - & $0,455^{*}$ & $0,535^{\star}$ \\
\hline Microcistina & $0,455^{*}$ & - & $0,387^{*}$ \\
\hline Clorofila-a & $0,535^{\star}$ & $0,387^{\star}$ & - \\
\hline Fósforo total & 0,004 & $0,232^{*}$ & $0,438^{*}$ \\
\hline Nitrogênio Amoniacal & $-0,309^{\star}$ & $-0,065$ & $-0,261^{*}$ \\
\hline Nitrato & 0,091 & 0,159 & 0,146 \\
\hline Nitrito & 0,005 & 0,107 & 0,051 \\
\hline DBO & $0,183^{*}$ & $0,258^{\star}$ & $0,648^{\star}$ \\
\hline Coliformes & $-0,413^{*}$ & $-0,066$ & $-0,091$ \\
\hline Termotolerantes ou E. coli & 0,091 & 0,027 & 0,083 \\
\hline Temperatura & $-0,061$ & - & - \\
\hline Microcistina/clorofila-a & - &
\end{tabular}

Os valores com * foram considerados significativos com nível de confiança de 95\%. o Jaguari não pode ter suas águas consideradas potencialmente tóxicas, pois apresentou concentrações de microcistina sempre inferiores a $0,16 \mu \mathrm{g} . \mathrm{L}^{-1}$ durante o período estudado.

\section{Conflitos entre os resultados do monitoramento de tais sistemas aquáticos e os seus respectivos enquadramentos}

Os cinco reservatórios de classe especial estudados foram comparados com os padrões estabelecidos para a classe 1 (Tabela 5). A comparação indicou que $47 \%$ dos dados dos primeiros apresentaram densidade de cianobactérias superior a 20.000 células. $\mathrm{mL}^{-1}$. A porcentagem de dados de clorofila-a superiores a $10 \mu \mathrm{g} . \mathrm{L}^{-1}$ foi de $74 \%$. Em relação aos dados de fósforo total, $73 \%$ foram superiores a $0,02 \mathrm{mg} \cdot \mathrm{L}^{-1}$. Em relação à DBO, houve incompatibilidade em aproximadamente metade (46\%) dos dados, com valores superiores a $3 \mathrm{mg} . \mathrm{L}^{-1}$ (Tabela 5). Os dois principais reservatórios que contribuíram para as incompatibilidades com o enquadramento, nesse caso, foram Billings e Jaguari.

Os corpos d'água enquadrados na classe especial têm suas águas destinadas, segundo a Resolução CONAMA nº 357/2005 (BRASIL, 2005), ao abastecimento para consumo humano, após desinfecção; à preservação do equilíbrio natural das comunidades aquáticas; e à preservação dos ambientes aquáticos em unidades de conservação de proteção integral. O relatório Qualidade das Águas Interiores no Estado de São Paulo de 2017 (CETESB, 2017) apontou que, entre os reservatórios enquadrados na classe especial, apenas dois (reservatórios Cabuçu e Jaguari) apresentaram valores anuais de IAP considerados "ótimos" ou "bons". O reservatório Guarapiranga foi classificado como "regular", enquanto o Jundiaí e o Billings tiveram suas águas brutas consideradas "ruins", pelo IAP, visando ao abastecimento público. Entende-se, dessa forma, que os reservatórios Jundiaí, Guarapiranga e Billings não estão atendendo aos critérios para a primeira destinação citada - abastecimento para consumo humano, após desinfecção - , uma vez que as águas provavelmente necessitam de tratamento convencional ou avançado para atingir os padrões de potabilidade.

Os dados dos reservatórios Itupararanga e Cascata (classe 2) foram incompatíveis com os limites para sua classe em 79\% dos casos em relação à densidade de cianobactérias. Cerca de $31 \%$ dos dados de clorofila-a ultrapassaram o padrão de qualidade estabelecido para essa mesma classe $\left(30 \mu \mathrm{g} . \mathrm{L}^{-1}\right)$. As incompatibilidades dos dados de fósforo total e DBO foram menores, 28 e 18\%, respectivamente. Conforme os dados da Tabela 2, o reservatório Cascata parece ser o maior responsável por tais não conformidades, uma vez que os valores médios dos parâmetros de suas águas são maiores que os encontrados no Itupararanga.

Cunha et al. (2013) estudaram as incompatibilidades de fósforo total e DBO nas mesmas UGRHI dos reservatórios Itupararanga (UGRHI 10) e Cascata (UGRHI 20) e obtiveram resultados semelhantes ou mais críticos aos observados neste estudo. Segundo os pesquisadores, as incompatibilidades 
de fósforo foram de 75 e $38 \%$ para as UGRHI 10 e 20, respectivamente, enquanto, para DBO, foram de 40 e $17 \%$, na mesma ordem.

No presente estudo, a situação mais crítica em relação às concentrações de microcistina foi observada no reservatório Cascata. Nesse caso, tais concentrações superaram o valor de referência de $1 \mu \mathrm{g} . \mathrm{L}^{-1} \mathrm{em}$ $65 \%$ das amostras, com valores de até $11 \mu \mathrm{g}$. $\mathrm{L}^{-1}$. As porcentagens relativamente elevadas de não conformidade com o enquadramento legal reforçam a necessidade de que seja resgatado o papel fundamental da Resolução CONAMA n 357/2005 (BRASIL, 2005), que é o de permitir o planejamento dos recursos hídricos e subsidiar metas progressivas de melhoria da qualidade da água, com vistas à manutenção dos usos múltiplos em longo prazo (CUNHA et al., 2013).

De maneira geral, por meio deste estudo, foi possível observar que ainda existe relativa falta de integração entre os programas de monitoramento de qualidade da água e a legislação em vigor no Brasil. Isso diz respeito não apenas ao monitoramento muitas vezes desacoplado de aspectos quantitativos (e.g., vazão) e qualitativos (parâmetros de qualidade da água) (MARQUES et al., 2019), mas também em relação às frequências de amostragem. Tais frequências (e.g., bimestral, mensal, semanal, diária) dependem principalmente dos custos vinculados à coleta e à análise das amostras (FIORE; BARDINI; NOVAES, 2017), da disponibilidade de recursos humanos e ainda da conveniência e de outros critérios subjetivos (STROBL; ROBILLARD, 2008). Sugere-se, assim, maior integração entre a Resolução CONAMA nº 274/2000, a Resolução CONAMA $n^{\circ}$ 357/2005 e Portaria de Consolidação nº 5/GM/MS (BRASIL, 2000; 2005 ; 2017), especialmente nos casos em que os mananciais possuem elevada variabilidade temporal, como aqueles localizados em bacias significativamente urbanizadas (ver mais detalhes em COELHO, 2013).

Existem algumas possibilidades para atenuar as divergências entre as frequências de amostragem dos programas de monitoramento e aquelas preconizadas pela legislação. Ferramentas estatísticas podem ser utilizadas para otimizar redes de monitoramento dos pontos de vista espacial e temporal (e.g., CALAZANS, 2015). Sensores automáticos para monitoramento de algumas variáveis da água também já vêm sendo utilizados para aumentar a resolução temporal das séries de dados de qualidade (ANTTILA et al., 2012), embora os custos ainda

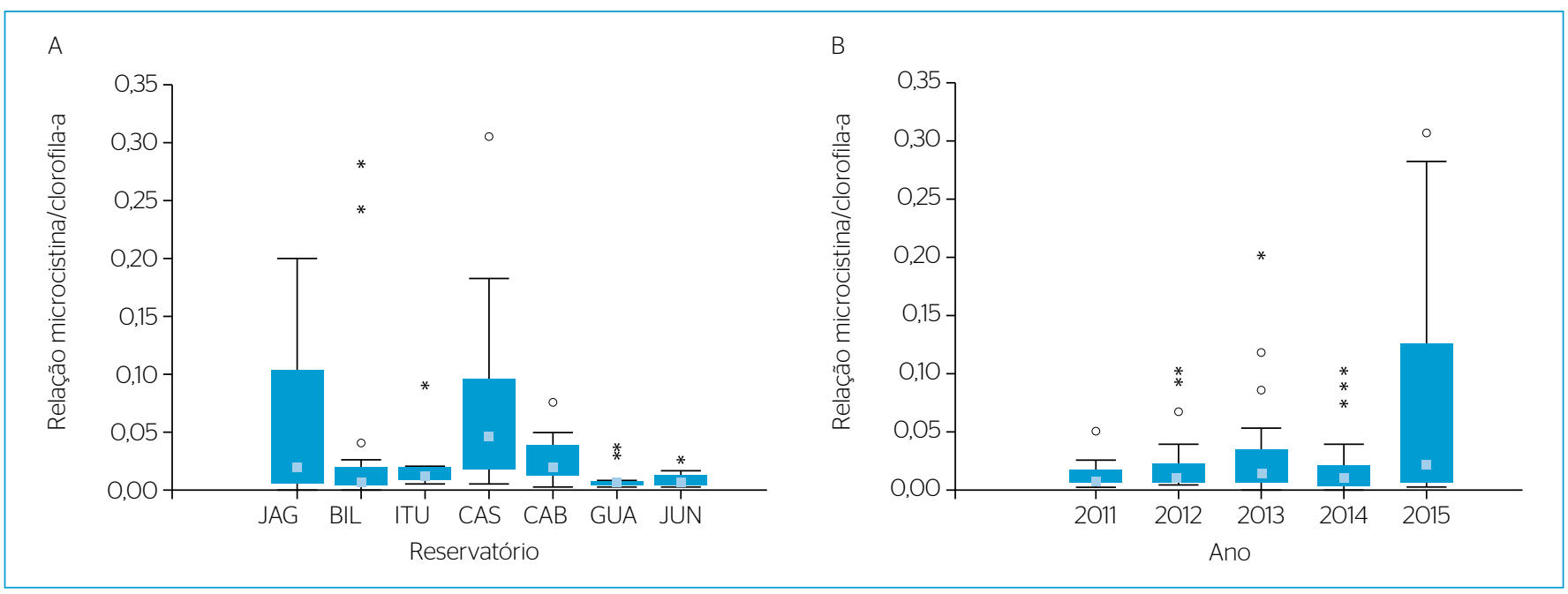

Figura 2 - Potencial tóxico das águas, representado pela relação microcistina/clorofila-a nos reservatórios do estado de São Paulo.

Tabela 5 - Valores máximos permitidos pelos padrões de qualidade e a porcentagem de não conformidade dos dados dos reservatórios em relação à Resolução CONAMA no 357/2005 (BRASIL, 2005).

\begin{tabular}{|c|c|c|c|c|}
\hline \multirow{2}{*}{ Variável } & \multicolumn{2}{|c|}{ Valor máximo } & \multicolumn{2}{|c|}{ Não conformidade dos reservatórios (\%) } \\
\hline & Classe 1 & Classe 2 & Classe especial* & Classe 2 \\
\hline Número de cianobactérias (células.mL'1) & 20.000 & 50.000 & 47 & 79 \\
\hline Clorofila-a $\left(\mu \mathrm{g} . \mathrm{L}^{-1}\right)$ & 10 & 30 & 74 & 31 \\
\hline Fósforo total (mg.L-1) & 0,02 & 0,03 & 73 & 28 \\
\hline Nitrogênio amoniacal (mg.L'1) & \multicolumn{2}{|c|}{0,5} & 2 & 8 \\
\hline Nitrato (mg. L-1) $^{-1}$ & \multicolumn{2}{|c|}{10} & $\mathrm{O}$ & $\mathrm{O}$ \\
\hline Nitrito $\left(m g . L^{-1}\right)$ & \multicolumn{2}{|c|}{1} & 0 & 0 \\
\hline Demanda bioquímica de oxigênio (mg.L¹) & 3 & 5 & 46 & 18 \\
\hline
\end{tabular}

*Foram considerados os critérios para classe 1. 
sejam, em geral, elevados e proibitivos para algumas agências de monitoramento. Tais ferramentas podem contribuir para o uso mais efetivo dos resultados de monitoramento para o gerenciamento dos recursos hídricos pelos tomadores de decisão (MIDAGLIA, 2011).

\section{CONCLUSÕES E RECOMENDAÇÕES}

A análise dos dados permitiu observar que ao menos quatro dos reservatórios estudados - Itupararanga, Guarapiranga, Cascata e Billings apresentaram, no período do estudo (2011 a 2015), elevadas densidades de cianobactérias. No entanto, apenas os dois últimos apresentam concentrações de microcistina preocupantes, acima do valor orientador recomendado pela Organização Mundial da Saúde (OMS) e pelo Anexo XX da Portaria de Consolidação n 5/GM/MS (BRASIL, 2017) para água tratada $-1 \mu \mathrm{g}$. $\mathrm{L}^{-1}$. Isso reforça a necessidade de monitoramento da água tratada e, eventualmente, de implantação de uma etapa de tratamento avançado para a remoção de cianotoxinas nas suas respectivas estações de tratamento de água. No que diz respeito ao número de coliformes termotolerantes ou E. coli, a maior parte dos dados apresentou valores menores que 250 e $200 \mathrm{UFC} .100 \mathrm{~mL}^{-1}$, respectivamente.

O presente estudo permitiu ainda verificar que o aspecto espacial (reservatório) teve maior influência sobre o número de células de cianobactérias, a microcistina e os coliformes termotolerantes ou $E$. coli que o aspecto temporal, o que sugere que as diferentes bacias de contribuição dos reservatórios afetam a qualidade da água de maneira diferenciada. Não foi observada tendência temporal de aumento ou diminuição desses parâmetros durante os anos avaliados.

As elevadas porcentagens de incompatibilidade dos dados de monitoramento dos reservatórios com os limites estabelecidos pelos padrões de qualidade reforçam a necessidade de melhoria da qualidade desses ambientes aquáticos para garantir os diversos usos, especialmente o abastecimento público. Porcentagens de não conformidade próximas a $80 \%$ para o número de cianobactérias, por exemplo, indicam a vulnerabilidade dos mananciais a episódios de florações tóxicas desses organismos e eventuais interferências na qualidade da água distribuída à população. $\mathrm{O}$ enquadramento dos cursos de água e os respectivos padrões de qualidade devem ser utilizados para fins de planejamento e para permitir o estabelecimento de metas progressivas de melhoria da qualidade da água, de modo que as classes menos restritivas sejam etapas intermediárias e de transição a uma situação mais adequada.

Por fim, esta pesquisa permitiu concluir que ainda existe, no Brasil, uma falta de integração entre as diferentes leis e resoluções relativas à qualidade das águas, por exemplo, em relação à uniformização das frequências de amostragem e monitoramento. Além disso, a presente análise mostrou que alguns dos reservatórios estudados, a despeito de serem utilizados para abastecimento público, possuem indicadores de contaminação e aporte significativo de matéria orgânica e outros poluentes. Ressalta-se, assim, a importância de investimentos na coleta e no tratamento de esgotos sanitários até níveis secundário e terciário para evitar o aporte excessivo de matéria orgânica e nutrientes aos mananciais. Recomenda-se ainda que as estações de tratamento que operam com as águas dos reservatórios Cascata possuam etapas avançadas para eventual necessidade de remoção de cianotoxinas.

\section{AGRADECIMENTOS}

Os autores agradecem ao Conselho Nacional de Desenvolvimento Científico e Tecnológico (CNPq) a bolsa de mestrado concedida ao primeiro autor, o auxílio financeiro (Processo $n^{\circ} 406855 / 2016-1$ ) e a bolsa de produtividade em pesquisa (Processo no 300899/2016-5) ao último autor. Agradecem também a dois revisores anônimos as pertinentes sugestões para aprimorar o artigo.

\section{REFERÊNCIAS}

AGÊNCIA NACIONAL DE ÁGUAS (ANA). (2013) Conjuntura dos recursos hídricos no Brasil: 2013. Brasília: ANA. 255 p.

ANTTILA, S.; KETOLA, M.; VAKKILAINEN, K., KAIRESALO, T. (2O12) Assessing temporal representativeness of water quality monitoring data. Journal of Environmental Monitoring, v. 14, p. 589-595. http:// doi.org/10.1039/C2EM10768F

AZEVEDO, S.M.F.O.; CARMICHAEL, W.W.; JOCHIMSEN, E.M.; RINEHART, K.L.; LAU, S.; SHAW, G.R.; EAGLESHAM, G.K. (2002) Human intoxication by microcystins during renal dialysis treatment in Caruaru - Brazil. Toxicology, v. 181-182, p. 441-446. https://doi. org/10.1016/s0300-483x(02)00491-2
BRASIL. (2000) Resolução CONAMA no 274, 29 de novembro de 2000. Dispõe sobre licenciamento ambiental; competência da União, Estados e Municípios; listagem de atividades sujeitas ao licenciamento; Estudos Ambientais, Estudo de Impacto Ambiental e Relatório de Impacto Ambiental. Diário Oficial [da] República Federativa do Brasil, Brasília.

BRASIL. (2005) Resolução CONAMA no 357, 17 de março de 2005. Dispõe sobre a classificação dos corpos de água e diretrizes ambientais para o seu enquadramento, bem como estabelece as condições e padrões de lançamento de efluentes, e dá outras providências. Diário Oficial [da] República Federativa do Brasil, Brasília. 
BRASIL. (2017) Portaria de Consolidação no 5/GM/MS, 28 de setembro de 2017. Dispõe, entre outros, sobre os procedimentos de controle e de vigilância da qualidade da água para consumo humano e seu padrão de potabilidade. Diário Oficial [da] República Federativa do Brasil, Brasília.

CALAZANS, G.M. (2015) Avaliação e Proposta de Adequação da Rede de Monitoramento da Qualidade das Águas Superficiais das Sub-Bacias do Rio das Velhas e do Rio Paraopeba Utilizando Técnicas Estatísticas Multivariadas. 196f. Dissertação (Mestrado) Escola de Engenharia, Universidade Federal de Minas Gerais.

CALIJURI, M.C.; ALVES, M.S.A.;SANTOS, A.C.A. (2006) Cianobactérias e cianotoxinas em águas continentais. São Carlos: Rima. 109 p.

CARVALHO, L.R.; SANT'ANNA, C.L.; GEMELGO, M.C.P.; AZEVEDO, M.T.P. (2007) Cyanobacterial occurrence and detection of microcystin by planar chromatography in surface water of Billings and Guarapiranga Reservoirs, SP, Brazil. Revista Brasileira de Botânica, v. 30, n. 1, p. 141-148. http://dx.doi.org/10.1590/S010084042007000100014

CHELLAPPA, N.T.; COSTA, M.A.A.M.; MARINHO, I.R. (2000) Harmful cyanobacterial blooms from semi-arid freshwater ecosystems of Northeast Brazil. Australian Society for Limnology, v. 38, p. 45-49.

CHORUS, I.; BARTRAM, J. (1999) Toxic cyanobacteria in water: a guide to their public health consequences, monitoring and management. Londres: E \& FN Spon. 400 p.

COELHO, M. (2013) Estratégia de monitoramento da qualidade da água para a gestão de recursos hídricos em bacias urbanas. $161 f$. Dissertação (Mestrado) - Universidade Federal do Paraná, Curitiba.

COMPANHIA AMBIENTAL DO ESTADO DE SÃO PAULO (CETESB). (2017) Qualidade das águas interiores no estado de São Paulo 2017. São Paulo: CETESB.

CORRELL, D.L. (1999) Phosphorus: a rate limiting nutrient in surface waters. Poultry Science, v. 78, n. 5, p. 674-682. https://doi. org/10.1093/ps/78.5.674

CUNHA, D.G.F.; CALIJURI, M. do C. (2011a) Limiting factors for phytoplankton growth in subtropical reservoirs: the effect of light and nutrient availability in different longitudinal compartments. Lake and Reservoir Management, v. 27, n. 2, p. 162-172. https://doi.or g/10.1080/07438141.2011.574974

CUNHA, D.G.F.; CALIJURI, M. do C. (2011b) Variação sazonal dos grupos funcionais fitoplanctônicos em braços de um reservatório tropical de usos múltiplos no estado de São Paulo (Brasil). Acta Botanica Brasilica, v. 25, n. 4, p. 822-831. http://dx.doi.org/10.1590/ S0102-33062011000400009

CUNHA, D.G.F.; CALIJURI, M. do C.; LAMPARELLI, M.C.; MENEGON, N. (2013) Resolução CONAMA 357/2005: análise espacial e temporal de não conformidades em rios e reservatórios do estado de São Paulo de acordo com seus enquadramentos (2005-2009). Engenharia Sanitária e Ambiental, v. 18, n. 2, p. 159-168. http://dx.doi. org/10.1590/S1413-41522013000200008
CUNHA, D.G.F.; GRULL, D.; DAMATO, M.; BLUM, J.R.; LUTTI, J.E.; EIGER, S.; MANCUSO, P.C.S (2011) Trophic state evolution in a subtropical reservoir over 34 years in response to different management procedures. Water Science and Technology, v. 64, n. 12, p. 2338-2344. https://doi.org/10.2166/wst.2011.826

CUNHA, D.G.F.; SABOGAL-PAZ, L.; DODDS, W.K. (2016) Land use influence on raw surface water quality and treatment costs for drinking supply in São Paulo State (Brazil). Ecological Engineering, v. 94, p. 516-524. http://dx.doi.org/10.1016/j.ecoleng.2016.06.063

DODDS, W.K.; PERKIN, J.S.; GERKEN, J.E. (2013) Human impact on freshwater ecosystem services: a global perspective. Environmental Science and Technology, v. 47, n. 16, p. 9061-9068. https://doi.org/10.1021/es4021052

ELSER, J.J.; BRACKEN, M.E.S.; CLELAND, E.E.; GRUNER, D.S.; HARPOLE, W.S.; HILLEBRAND, H.; NGAI, J.T.; SEABLOOM, E.W.; SHURIN, J.B.; SMITH, J.E. (2007) Global analysis of nitrogen and phosphorus limitation of primary producers in freshwater, marine and terrestrial ecosystems. Ecology Letters, v. 10, n. 12, p. 1135-1142. https://doi.org/10.1111/j.1461-0248.2007.01113.x

FIORE, F.A.; BARDINI, V.S.S.; NOVAES, R.C. (2017) Monitoramento da qualidade de águas em programas de pagamento por serviços ambientais hídricos: estudo de caso no município de São José dos Campos/SP. Engenharia Sanitária e Ambiental, v. 22, n. 6, p. 1141-1150. http://dx.doi.org/10.1590/s1413-41522017165072

GUEDES, I.A.; COSTA LEITE, D.M.; MANHÃES, L.A.; BISCH, P.M.; AZEVEDO, S.M.F.O.; PACHECO, A.B.F. (2014) Fluctuations in microcystin concentrations, potentially toxic Microcystis and genotype diversity in a cyanobacterial community from a tropical reservoir. Harmful Algae, v. 39, p. 303-309. http://dx.doi.org/10.1016/j.hal.2014.09.001

HALL, T.; HART, J.; CROLL, B.; GREGORY, R. (2000) Laboratory-scale investigations of algal toxin removal by water treatment. Journal of the Chartered Institution of Water and Environmental Management, v. 14, n. 2, p. 143-149. https://doi.org/10.1111/j.1747-6593.2000.tb00241.x

HIMBERG, K.; KEIJOLA, A.M.M.; HIISVIRTA, L.; PYYSALO, H.; SIVONEN, K. (1989) The effect of water treatment processes on the removal of hepatotoxins from Microcystis and Oscillatoria cyanobacteria: A laboratory study. Water Research, v. 23, n. 8, p. 979-984. https://doi.org/10.1016/0043-1354(89)90171-1

HIROOKA, E.Y.; PINOTTI,M.H.P.; TSUTSUMI,T:;YSHIDA, F.;UENO,Y.(1999) Survey of Microcystins in water between 1995 and 1996 in Paraná, Brazil using ELISA. Natural Toxins, v. 7. n. 3, p. 103-109. https://doi.org/101002/ (SICI)1522-7189(199905/06)7:3\%3C103:AID-NT47\%3E3.0.CO;2-D

INSTITUTO BRASILEIRO DE GEOGRAFIA E ESTATÍSTICA (IBGE) (2008) Pesquisa Nacional de Saneamento Básico do ano de 2008. Disponível em: <https://sidra.ibge.gov.br/pesquisa/pnsb>. Acesso em: 20 fev. 2018.

KOTAK, B.G.; LAM, A.K.Y.; PREPAS, E.E.; HRUDEY, S.E. (2000) Role of chemical and physical variables in regulating microcystin-LR concentration in phytoplankton of eutrophic lakes. Canadian Journal of Fisheries and Aquatic Sciences, v. 57, n. 8, p. 1584-1593. http://dx.doi.org/10.1139/cjfas-57-8-1584 
LOFTIN, K.A.; GRAHAM, J.L.; HILBORN, E.D.; LEHMANN, S.C.; MEYER, M.T.; DIETZE, J.E.; GRIFFITH, C.B. (2016) Cyanotoxins in inland lakes of the United States: occurrence and potential recreational health risks in the EPA National Lakes Assessment 2007. Harmful Algae, v. 56, p. 77-90. https://doi.org/10.1016/j.hal.2016.04.001

LOPES, A.M.M.B.; GOMES, L.N.L.; MARTINS, F.C.; CERQUEIRA, D.A.; MOTA FILHO, C.R.; VON SPERLING, E.; PÁDUA, V.L. (2017) Dinâmica de protozoários patogênicos e cianobactérias em um reservatório de abastecimento público de água no sudeste do Brasil. Engenharia Sanitária e Ambiental, v. 22, n. 1, p. 25-43. http:// dx.doi.org/10.1590/s1413-41522016143529

MARQUES, L.O.A.; TAFFARELLO, D.; CALIJURI, M.C.; MENDIONDO, E.M.; FERREIRA, M.S.; CUNHA, D.G.F. (2O19) Phosphorus and thermotolerant coliforms' loads in Brazilian watersheds with limited data: considerations on the integrated analysis of water quality and quantity. Revista Brasileira de Recursos Hidricos, v.24. http://doi.org/10.1590/2318-0331.241920170137

MATTHIENSEN, A.; BEATTIE, K.A.; YUNES, J.S.; KAYA, K.; CODD, G.A. (2000) [d-Leu1]Microcystin-LR, from the cyanobacterium Microcystis RST 9501 and from a Microcystis bloom in the Patos Lagoon estuary, Brazil. Phytochemistry, v. 55, n. 5, p. 383-387. https:// doi.org/10.1016/s0031-9422(00)00335-6

MEREL, S.; WALKER, D.; CHICANA, R.; SNYDER, S.; BAURES, E.; THOMAS, O. (2O13) State of knowledge and concerns on cyanobacterial blooms and cyanotoxins. Environment International, v. 59, p. 303-327. https://doi.org/10.1016/j.envint.2013.06.013

MIDAGLIA, C.V.L. (2011) Proposta de implantação do indice de abrangência espacial de monitoramento-IAEM por meio da análise da evolução da rede de qualidade das águas superficiais do estado de São Paulo. 229f. Tese (Doutorado) - Universidade de São Paulo, São Paulo.

MOSCHINI-CARLOS, V.; BORTOLI, S.; PINTO, E.; NISHIMURA, P.Y; FREITAS, L.G.; POMPEO, M.L.M.; DÖRR, F. (2009) Cyanobacteria and cyanotoxin in the billings reservoir (São Paulo, SP, Brazil). Limnetica, v. 28, n. 2, p. 273-282. http://doi.org/10.23818/limn.28.23

OH, H.M.; LEE, S.J.; JANG, M.-H.; YOON, B.-D. (2000) Microcystin production by Microcystis aeruginosa in a phosphorous-limited chemostat. Applied and Environmental Microbiology, v. 66, n. 1, p. 176-179. http://doi.org/10.1128/AEM.66.1.176-179.2000

PAIVA, R.F.P.S.; SOUZA, M.F.P. (2018) Associação entre condições socioeconômicas, sanitárias e de atenção básica e a morbidade hospitalar por doenças de veiculação hídrica no Brasil. Cadernos de Saúde Pública, v. 34, n. 1, p. e00017316. http://dx.doi. org/10.1590/0102-311×00017316

SCHINDLER, D.W.; HECKY, R.E.; FINDLAY, D.L.; STAINTON, M.P.; PARKER, B.R.; PATERSON, M.J.; BEATY, K.G.; LYNG, M.; KASIAN, S.E.M. (2008) Eutrophication of lakes cannot be controlled by reducing nitrogen input: Results of a 37-year whole-ecosystem experiment. Proceedings of the National Academy of Sciences, v. 105, n. 32, p. 11254-11258. https://doi.org/10.1073/pnas.0805108105

STROBL, R.O.; ROBILLARD, P.D. (2008) Network design for water quality monitoring of surface freshwaters: A review. Journal of Environmental Management, v. 87, n. 4, p. 639-648. https://doi. org/10.1016/j.jenvman.2007.03.001

SVIRČEV, Z:; DROBAC, D.; TOKODI, N.; MIJOVIĆ, B.; CODD, G.A.; MERILUOTO, J. (2017) Toxicology of microcystins with reference to cases of human intoxications and epidemiological investigations of exposures to cyanobacteria and cyanotoxins. Archives of Toxicology, v. 91, n. 2, p. 621-650. https://doi.org/10.1007/s00204016-1921-6

TEIXEIRA, M.D.G.; COSTA, M.D.C.; CARVALHO, V.L.; PEREIRA, M.D.S.; HAGE, E. (1993) Epidemia de gastroenterite na área de barragem de Itaparica, Bahia. Bulettin of Sanitary Panamerican, v. 114, n. 6, p. $502-512$

VÖRÖSMARTY, C.J.; MCINTYRE, P.B.; GESSNER, M.O; DUDGEON, D.; PRUSEVICH, A.; GREEN, P.; GLIDDEN, S.; BUNN, S.E.; SULLIVAN, C.A.; REIDY LIERMANN, C.; DAVIES, P.M. (2O10) Global threats to human water security and river biodiversity. Nature, v. 467, p. 555561. https://doi.org/10.1038/nature09440

WOOD, R. (2016) Acute animal and human poisonings from cyanotoxin exposure - A review of the literature. Environment International, v. 91, p. 276-282, 2016. http://dx.doi.org/10.1016/j. envint.2016.02.026 